\title{
Patients as equal partners in decision-making: The global reality
}

\author{
Anubha Taneja Mukherjee \\ Director, Public Affairs \& Advocacy, Genesis Burson-Marsteller; Member Secretary, India \\ Thalassemia Patients Advocacy Group; Joint Secretary, Thalassemics India
}

Decision making is an inherently complicated procedure, which by its very nature requires the decision-maker to co-opt all the stakeholders concerned. The procedure of decision-making may vary from country to country, depending on its size, culture, history and special demographic circumstances. Around the world, key decision-makers include the executive, the legislature and the judiciary. While the distribution of powers between these three may vary in tandem with their relation to each other, their roles remain the same. While the legislature enacts laws for its citizens, the executive, popularly known as the government, implements these laws and while doing so promulgates policies that are in alignment with the said laws. Mostly, the executive is also authorised to promulgate some laws of its own. The judiciary, on the other hand, comes into the picture when there is a dispute with regard to such laws. It also steps in on its own at times. While settling such disputes, the judiciary also ends up setting what we know as precedents, which also become a part of the legal fabric of a society. In a nutshell, these three are the key decision makers in any country.

As mentioned above, while making decisions, these authorities are mostly required to co-opt all the stakeholders concerned, thereby making decision making a consultative process. These stakeholders include think tanks, research bodies, media and most importantly the affected party. The reason for having such a consultative procedure in place is that the decision makers are not experts in every subject or issue that comes their way. For instance, when a need to promulgate a national policy on thalassemia presents itself to a certain government, whether it be owing to media reportage or representations from the civil society, the decision makers will look towards people considered to be the experts in the subject to come forward and be a part of the policy making. One could say that this sounds like an ideal situation where the government actually invites people concerned with thalassemia to come forward and share views about it for the purpose of policy making. It is, however, true! It is as true for India as it is for any developed country. What we must ensure then is that the government or the decision maker considers us, the patients, as the experts. While it does sound obvious that those impacted with the disorder would be the ones with the first-hand knowledge about the disorder, the very fact that there is a topic in this conference on the role of patients in decision making speaks volumes about the distance that remains to be covered by the patients of thalassemia as far as participation in decision-making is concerned.

With the massive strides in the field of medical science and the unflinching support of organisations like Thalassemia International Federation (TIF), we have now reached the stage where we must step out of the victim mode and represent ourselves before the decision-makers, whether by forming Patients Advocacy Groups or otherwise. One may take cue from various associations around the world. Global HD Organisations are a good example. They are known to have got together to give patients a voice in clinical research. The most popular strategy for reaching out to the decision makers is to unite, engage, and partner both in private meetings and consultative fora like events, task forces and projects. "Unite, Engage \& Partner" can therefore be the most successful mantra for engaging with the decision makers.

Talking of examples of advocacy and participation by patients, while there are numerous examples in Europe and North America of the power of patient advocacy so much so that patients are on the same level as doctors when it comes to voicing opinions in policy making, TIF on an international level has created since 2009 the Expert Patients Programme, and is now moving forward in giving patients a voice through its educational platform. Recently, India also launched its first Thalassemia Patients Advocacy Group (PAG) in the august presence of the Deputy Chief Minister of the capital of the country. The India PAG has seven patients from the fields of law, psychology, education and IT. The Group is already involved with the government on the formulation of the National Thalassemia Policy. This is a great start and this should give enough and more encouragement to thalassemics across the world to UNITE, ENGAGE AND PARTNER in the process that impacts them the most - decision-making!
Correspondence: Anubha Taneja Mukherjee.

E-mail: anubhaarjun@gmail.com

This work is licensed under a Creative Commons Attribution 4.0 License (by-nc 4.0).

CCopyright A. Taneja Mukherjee, 2018

Licensee PAGEPress, Italy

Thalassemia Reports 2018; 8:7472

doi:10.4081/thal.2018.7472 